\title{
Case-control study of vulvar vestibulitis risk associated with genital infections
}

\author{
Elaine M. Smith ${ }^{1,2}$, Justine M. Ritchie ${ }^{3}$, Rudolph Galask ${ }^{2}$, \\ Erica E. Pugh ${ }^{1}$, Jian Jia ${ }^{3}$ and Joan Ricks-McGillan ${ }^{1}$ \\ ${ }^{1}$ Department of Epidemiology, College of Public Health, \\ ${ }^{2}$ Department of Obstetrics/Gynecology, College of Medicine and \\ ${ }^{3}$ Department of Biostatistics, College of Public Health, University of Iowa, Iowa City, IA
}

Objective: To evaluate the risk of vulvar vestibulitis syndrome (WS) associated with genital infections in a case-control study.

Methods: Diagnosed cases with VVS $(n=69)$ and age-frequency-matched healthy controls $(n=65)$ were enrolled from gynecology clinics in a university medical hospital during 1999. They were compared for potential risk factors and symptoms of disease.

Results: VVS cases had a significantly higher risk of physician-reported bacterial vaginosis (BV) (odds ratio, $O R=9.4)$, Candida albicans ( $O R=5.7)$, pelvic inflammatory disease (PID) $(O R=I I .2)$, trichomoniasis $(O R=20.6)$, and vulvar dysplasia $(O R=15.7)$ but no risk associated with human papillomavirus (HPV), ASCUS, cervical dysplasia, genital warts, chlamydia, genital herpes or gonorrhea. Genital symptoms reported significantly more often with VVS included vulvar burning ( 91 vs. $12 \%)$, dyspareunia (8I vs. 15\%), vulvar itching (68 vs. $23 \%$ ) and dysuria (54 vs. 19\%) $(p<0.000 \mathrm{I})$.

Conclusion: A history of genital infections is associated with an increased risk of VVS. Long-term follow-up case-control studies are needed to elucidate etiologic mechanisms, methods for prevention and effective treatment.

Key words: Vulvar Vestibulitis; Genital Infections; Risk Factors; Vestibulitis Symptoms

The prevalence of vulvar vestibulitis syndrome (VVS) has been estimated to affect approximately $15 \%$ of the adult female population who are seen in gynecologic practices ${ }^{1,2}$. The condition has been defined by the International Society for the Study of Vulvar Disease (ISSVD) according to the following criteria: (1) severe pain on vestibular touch or vaginal entry; (2) tenderness on pressure localized within the vulvar vestibule; and (3) physical findings of erythema confined to the vestibule ${ }^{2,3}$. VVS is an inflammatory process of the vestibule of the vagina that involves the mucous membrane and its underlying appendages, the vestibular ducts and glands. Vestibular pain may develop gradually or suddenly, followed by pain-free periods ${ }^{1,4}$.

Characterized as the most frequent cause of dyspareunia, VVS is under-diagnosed, multi-factorial and complex. Patients present with unexplained pain, burning, stinging and rawness localized to the vulvar area ${ }^{1,5}$. Vestibulitis patients often are misdiagnosed with a psychological condition rather than physically based disease ${ }^{1}$. Previous studies have provided inconclusive evidence into

Correspondence to: Elaine M. Smith, MPH, PhD, Departments of Epidemiology and Biostatistics, College of Public Health, University of Iowa, Iowa City, IA 52242. Email: elaine-smith@uiowa.edu 
the etiology of VVS. Some studies propose that genital infections such as chronic candidiasis, herpes simplex virus (HSV), human papillomavirus (HPV), contact dermatitis, irritants or vulvar trauma cause VVS, but the findings have been contradictory $^{6-12}$. It has been postulated that specific microflora may induce injury to the vestibular nerve endings that leads to the extreme pain as well as alterations in the immune system ${ }^{13,14}$. Several studies also have suggested that there is a hormonal component that increases the risk of developing the syndrome possibly by altering the quality or quantity of mucus secretions in the vestibular glands, reducing the protective effect of the vestibule ${ }^{10}$. The purpose of this epidemiologic study was to evaluate the role of pathologic vaginal microbial and viral agents as risk factors for VVS among women diagnosed in a vulvar disease clinic and a group of healthy women without this diagnosis at a university hospital tertiary care center.

\section{METHODS}

\section{Population}

Patients with VVS were recruited from the Vulvar Disease Clinic at the University of Iowa Hospitals and Clinics (UIHC). The clinic sees in excess of 2500 patients with vulvar and vaginal complaints each year. Cases were recruited during two time-periods between August and September and October and November 1999. Patients were diagnosed as having VVS if they had the ISSVD criteria as described above. Since not all of the patients were newly diagnosed with the disease, they did not all currently have all these criteria at the time of interview. One woman was excluded because she did not complete the questionnaire, providing a sample size of 69 cases ( $98.6 \%$ participation). Between December 1999 and May 2000, a control group of women who had never had a diagnosis or any symptoms of VVS were solicited from general gynecology clinics in the UIHC $(n=65)$ where they were having annual exams. They were compared with the VVS cases for risk-factor history (98.5\% participation). A single physician who has diagnosed and treated the condition for approximately 20 years at the University of Iowa clinics diagnosed all cases (Rudolph Galask). He and two other physicians in the obstetrics/gynecology department served as resources for recruiting control subjects who were seeking routine gynecologic care. The controls were matched to cases based on age-frequency at 2 -year intervals. At the time of enrollment, controls were diagnosed as being free of VVS and having no prior history of the condition. Patients signed an informed consent form (approved by the Human Subjects Review Board that oversees medical research at the university) prior to enrolling in the study and completing the questionnaire. One case and one control declined participation in the study; thus the final sample size included 69 VVS cases and 65 controls.

\section{Procedures}

With the exception of Pap smears and HPV DNA testing, the diagnoses of genital infections and related diseases were those from the referring physician and a review of the medical chart among those who were patients of the university hospital. HPV testing was performed at the time of their clinic and enrollment into the study. Patients underwent one additional swab to test for HPV DNA during their routine gynecological exam. The HPV specimen was collected immediately following the Pap smear using a cotton tip applicator. Cells from the vulvar and vaginal region were placed in a sterile tube of saline, centrifuged and frozen at $-70^{\circ} \mathrm{C}$ for storage until the laboratory assessment was conducted. The HPV DNA specimens were evaluated by PCR amplification followed by DNA sequencing in the HPV labs where these tests are performed routinely using standardized techniques for other research protocols ${ }^{15}$.

\section{Questionnaire and genital infection reports}

Patients completed a 10-minute self-administered risk-factor questionnaire at the time of enrollment in the study. The research staff reviewed the questionnaire and queried unanswered questions. Information focused on sociodemographic characteristics, sexual behavior and pregnancy history, steroid hormone use (oral contraceptives (OCs) and other sex steroid hormones), gynecological history, alcohol and smoking exposure, history of 
genital infection and symptoms related to VVS and its treatment. Responses were validated using the patients' medical records. Cases and controls completed the same questionnaire except controls omitted sections related to some of the VVS symptoms. The research personnel completed a gynecological history from which they elicited information to verify a diagnosis and date of VVS, gynecologic treatment for the disease, irritants of VVS and other gynecological history to substantiate patient statements about steroid hormone use, sexual history, disease symptoms, genital infections, history of genital warts, abnormal Pap smears, genital dysplasia and invasive cancer, and other genital conditions or diseases. The basis for reporting vulvar intraepithelial neoplasia (VIN) was from the university pathology report and that of the gynecologist pathologist at the hospital. Candidiasis was evaluated separately from reports of yeast infection using the medical record since this specific infection has been the focus of several studies of VVS.

\section{Data analysis}

Comparisons between cases and controls for ontinuous variables were examined using the Wilcoxon rank sum test. Significance of risk factors after adjusting for age was based on the Wald statistics from the unconditional logistic regression models. Ninety-five percent confidence intervals (CI) for age-adjusted odds ratios (OR) for disease were calculated using the standard errors from the corresponding logistic regression models and the normal approximation. In comparisons where there was a single zero cell count, age-adjusted analyses were not performed. Instead, the Fisher's exact test was used to test the association between vestibulitis and the genital infections and a correction of $0.5^{16}$ was added to each cell count to estimate the crude OR for disease and the corresponding 95\% CI. All calculations were conducted using the SAS statistical package ${ }^{17}$.

\section{RESULTS}

\section{Demographic characteristics}

Characteristics of the study population are presented in Table 1. VVS cases were somewhat younger on average than were controls. There was no statistically significant difference in income or educational levels between cases and controls although diagnosis of VVS was more likely to occur with increasing income (Table 1). Compared with married women, other marital status groups were actually at lower risk of VVS. There was no association between smoking or alcohol and risk of disease. All of the cases and $92 \%$ of the controls were non-Hispanic white (data not shown).

\section{Sexual behavior/hormone use}

Most patients were heterosexual (97\% cases vs. $92 \%$ controls) with a few bisexual (3 vs. $4 \%$ ) or homosexual (0 vs. $4 \%)$. Among cases, $6.1 \%(n=4)$ stated that they never had sexual intercourse compared to $4 \%(n=1)$ of controls. Few VVS women $(12 \%)$ had changed sexual partners since the onset of their symptoms. The majority of partners reported no symptoms such as itching, irritation, burning or other indication of an infectious agent. The mean age at first intercourse was similar between cases and controls (Table 2). Age-adjusted risks indicate that older age at first intercourse increased the risk of VVS more than twofold. Nulliparity also was associated with a significant increased risk whereas use of OCs showed a nonsignificant protective effect against VVS. Nine of 29 parous VVS cases had symptoms prior to and during pregnancy. Use of sex hormones other than OCs also was associated with a slightly elevated nonsignificant risk of disease.

\section{Genital infections}

Most of the infectious diseases (Table 3) were reported more frequently in the VVS group. Exceptions included a history of ASCUS and cervical dysplasia, which are known to be increased in the presence of past and current HPV infection. In contrast, reports of a prior diagnosis of vulvar dysplasia, which is associated with HPV infection in younger-aged women, were found in $10 \%$ of the case group but not at all in controls. A history of genital warts, which is caused by non-oncogenic HPV types, was reported slightly more frequently in VVS cases but the difference 
Table I Demographics, sexual behavior and physician-diagnosed genital diseases of VVS cases and controls

\begin{tabular}{|c|c|c|c|c|}
\hline Demographic characteristic & Cases $(\mathrm{n}=69)$ & Controls $(\mathrm{n}=65)$ & $\mathrm{p}$-value & Age-adjusted OR $(95 \% \mathrm{Cl})$ \\
\hline Mean age, years (range) & $33(19-63)$ & $36(2 \mid-65)$ & 0.05 & - \\
\hline Mean education, years (range) & $15.3(|0-2|)$ & $15.9(|2-2|)$ & 0.19 & - \\
\hline \multicolumn{5}{|l|}{ Income, $n(\%)$} \\
\hline$<\$ 20000$ & $9(13)$ & $10(15.4)$ & - & I \\
\hline$\$ 20000-39999$ & $17(24.6)$ & $22(33.9)$ & 0.68 & $1.3(0.4-4)$ \\
\hline$\$ 40000-59000$ & $21(30.4)$ & $15(23.1)$ & 0.08 & $3.1(0.9-10.7)$ \\
\hline$\geq \$ 60000$ & $20(29)$ & $18(27.7)$ & 0.08 & $3.3(0.9-12.1)$ \\
\hline \multicolumn{5}{|l|}{ Marital status, $n(\%)$} \\
\hline Married & $47(68.1)$ & $38(58.5)$ & - & I \\
\hline Single & $17(24.6)$ & $16(24.6)$ & 0.2 & $0.6(0.2-1.4)$ \\
\hline Separated/divorced & $5(7.3)$ & II (16.9) & 0.054 & $0.3(0.1-1)$ \\
\hline \multicolumn{5}{|l|}{ Smoking, n (\%) } \\
\hline Never & $53(76.8)$ & $46(70.8)$ & - & I \\
\hline Given up & $10(14.5)$ & $9(13.9)$ & 0.73 & $1.2(0.4-3.3)$ \\
\hline Current & $6(8.7)$ & $10(15.4)$ & 0.31 & $0.6(0.2-1.7)$ \\
\hline \multicolumn{5}{|l|}{ Alcohol, $n(\%)$} \\
\hline Never & $35(50.7)$ & $22(33.8)$ & - & I \\
\hline Given up & $12(17.4)$ & I5 (23.I) & 0.93 & I (0.4-2.5) \\
\hline Current & $22(31.9)$ & $28(43.1)$ & 0.09 & $0.5(0.2-I . I)$ \\
\hline
\end{tabular}

$\mathrm{Cl}$, confidence interval; OR, odds ratio; WS, vulvar vestibulitis syndrome

Table 2 Sexual behavior and sex steroid hormone use of WS cases and controls

\begin{tabular}{lcccc}
\hline Sexual behavior/hormone use & Cases & Controls & p-value & Age-adjusted OR (95\% CI) \\
\hline $\begin{array}{l}\text { Mean age at first intercourse, } \\
\text { years (range) }\end{array}$ & $18.9(14-28)$ & $18.2(13-30)$ & 0.18 & - \\
& $\mathrm{n}(\%)$ & $\mathrm{n}(\%)$ & & \\
$<18$ & $22(34.9)$ & $30(50)$ & - & $\mathrm{I}$ \\
$\geq 18$ & $4 \mathrm{I}(65.1)$ & $30(50)$ & 0.02 & $2.3(1.1-4.9)$ \\
Currently having sex & $57(87.7)$ & $49(77.8)$ & 0.33 & $1.5(0.7-3.6)$ \\
Number of pregnancies & & & & $3.3(1.4-7.6)$ \\
0 & $40(58)$ & $19(29.2)$ & $<0.01$ & 1 \\
$\geq 1$ & $29(42)$ & $46(70.8)$ & - & 1 \\
OC use & $9(13)$ & $5(7.7)$ & - & $0.4(0.1-1.2)$ \\
$\quad$ Never & $26(37.7)$ & $39(60)$ & 0.1 & $0.7(0.2-2.8)$ \\
No longer & $34(49.3)$ & $21(32.3)$ & 0.67 & $1.7(0.8-4)$ \\
$\quad$ Current & $22(31.9)$ & $18(27.7)$ & 0.19 & \\
Use of other hormones & & & & \\
\end{tabular}

$\mathrm{Cl}$, confidence interval; OC, oral contraceptive; OR, odds ratio; WS, vulvar vestibulitis syndrome

was not statistically significant. However, there was no association between current HPV status and historical reporting of these HPV-related diseases. Furthermore, none of the patients had an
HPV-related disease at the time of enrollment. A number of other microflora were also associated with an increased risk of vestibulitis: candidiasis, trichomoniasis, bacterial vaginosis $(\mathrm{BV})$ and pelvic 
Table 3 Physician-diagnosed genital infections and other medical conditions of WS cases and controls

\begin{tabular}{|c|c|c|c|c|}
\hline Medical condition & Cases n (\%) & Controls n (\%) & p-value & Age-adjusted OR (95\% Cl) \\
\hline \multicolumn{5}{|l|}{ Genital infections/diseases } \\
\hline HPV infection & $18(26.1)$ & I8 (27.7) & 0.75 & $0.9(0.4-1.9)$ \\
\hline ASCUS & $10(14.5)$ & $17(26.2)$ & 0.09 & $0.5(0.2-1.1)$ \\
\hline Cervical dysplasia & II(I5.9) & $18(27.7)$ & 0.1 & $0.5(0.2-1.2)$ \\
\hline Vulvar dysplasia* & $7(10.1)$ & $0(0)$ & 0.01 & $15.7(0.9-28 I)$ \\
\hline Vaginal dysplasia & $0(0)$ & $0(0)$ & - & - \\
\hline Genital cancer & $0(0)$ & $0(0)$ & - & - \\
\hline Genital warts & $7(10.1)$ & $4(6.2)$ & 0.4 & $1.9(0.5-6.9)$ \\
\hline Yeast infection* & $29(42)$ & $0(0)$ & $<0.0001$ & $95.4(5.7-1604)$ \\
\hline Candidiasis & $15(21.7)$ & $3(4.6)$ & $<0.01$ & $5.7(I .5-2 \mid)$ \\
\hline Trichomoniasis* & $9(13)$ & $0(0)$ & $<0.01$ & $20.6(I .2-36 I)$ \\
\hline Bacterial vaginosis & $8(11.6)$ & $\mathrm{I}(\mathrm{I} .5)$ & 0.03 & $9.4(1.1-79)$ \\
\hline Chlamydia & $6(8.7)$ & $6(9.2)$ & 0.91 & I $(0.3-3.3)$ \\
\hline Pelvic inflammatory disease* & $5(7.3)$ & $0(0)$ & 0.06 & $11.2(0.6-206)$ \\
\hline Genital herpes & $3(4.4)$ & $5(7.7)$ & 0.48 & $0.6(0.1-2.6)$ \\
\hline Gonorrhea & $\mathrm{I}(\mathrm{I} .5)$ & $2(3.1)$ & 0.61 & $0.6(0.05-7)$ \\
\hline Syphilis & $0(0)$ & $0(0)$ & - & - \\
\hline \multicolumn{5}{|l|}{ Other conditions } \\
\hline Upper respiratory infections & $29(42)$ & $15(23.1)$ & 0.03 & $2.4(1.1-5)$ \\
\hline Muscle aches & $21(30.4)$ & $8(12.3)$ & $<0.01$ & $3.8(1.5-9.9)$ \\
\hline Immunodeficiency disease* & $4(5.8)$ & 0 & 0.12 & $9(0.5-170)$ \\
\hline Increased tiredness & $27(39.1)$ & $7(10.8)$ & $<0.01$ & $5.6(2.2-14.4)$ \\
\hline Poor wound healing & $4(5.8)$ & $\mathrm{I}(\mathrm{I} .5)$ & 0.21 & $4.3(0.4-40.6)$ \\
\hline
\end{tabular}

*Adjusted for 0.5 correction factor due to ' 0 ' count in cell - age-adjusted rates could not be determined; $\mathrm{Cl}$, confidence interval; HPV, human paillomavirus; OR, odds ratio; WS, vulvar vestibulitis syndrome

inflammatory disease (PID). There was no difference in risk between VVS cases and controls for gonorrhea, syphilis or vaginal dysplasia.

\section{Other medical conditions}

Patients were queried about some other diseases that have been reportedly higher in VVS patients, and which may be related to immunologic alterations or other unknown factors causative in the development of vestibulitis. The findings suggest that most of these were found more often in VVS cases than in controls: upper respiratory infections (URIs), muscle aches not related to some other specific medical condition, immunodeficiency diseases, increased tiredness and poor wound healing (Table 3).

\section{Symptom characteristics}

On average, approximately 2 years passed between the time of initial symptoms and a correct diagnosis of the disease. The average time between diagnosis and time of interview among the participating cases was 4.9 years. Only $21 \%$ of the cases were diagnosed within 6 months and $36 \%$ within 1 year. After 3 years, $75 \%$ of cases had received a correct clinical diagnosis but it was not uncommon for patients to remain undiagnosed for 12 or more years. At the time of enrollment in the study, which would have occurred several years after initial diagnosis, patients were asked to describe their current symptom status. Over half (53\%) of the patients had intermittent symptoms. A large percentage $(43 \%)$ still had constant, persistent symptoms compared with the time of diagnosis 
but they felt better than at the onset of the initial symptoms. A small number of women reported suffering constant symptoms that never improved (Table 4).

Compared with women without the disease, VVS cases at the time of interview were more likely to report multiple genital symptoms with the exception of rectal pain (Table 4). All VVS patients reported some pain at the time of diagnosis. The most frequently cited symptoms in the VVS patients were vulvar burning (91\%) and dyspareunia (81\%) whereas the most frequently reported symptom in controls was that of vulvar itching (23\%). Other symptoms specifically associated with VVS included discharge, which was most frequently white (55\%), clear (40\%) or yellow (40\%) in color. During symptomatic periods, $93.3 \%$ of cases had discharge while during non-symptomatic times, $69.6 \%$ still reported some discharge. The consistency of the discharge during symptomatic periods varied within patients and was most often reported as mucosy (70\%), watery (37\%) and/or cheesy (12\%). Most cases described the amount of discharge from light (52\%) to moderate $(40 \%)$ and many noticed an odor to the discharge (43\%). Among those who were receiving treatment for VVS at the time of enrollment, the most common drugs used were an A\&D ointment (25\%), nystatin-triamcinolone ointment/ mycolog (23\%), baking soda soaks (12\%), triamcinolone ointment (8\%) and hydrocortisone ointment (8\%). None of the patients received surgical treatment.

\section{Incident versus prevalent cases}

Women who were considered incident cases (21\%) based on having initial symptoms and diagnosis within 6 months showed some differences in symptomotology compared to the prevalent cases (79\%). There were few differences between the incident and prevalent cases by demographic or sexual behavior characteristics previously described in Tables 1 and 2. Newly diagnosed cases were more likely than previously diagnosed women to be former (67 vs. $17 \%, p<0.01$ ), but not current, alcohol users. Incident cases were less likely to have been aged 18 years or older at first intercourse (25 vs. $35 \%$ ) and more likely to be current users of OCs (64 vs. 48\%), but the significance in relation to disease is unclear. Incident cases were more frequently told they had vulvar dysplasia (21 vs. 8\%), and cervical dysplasia (21 vs. $15 \%)$ but were less commonly diagnosed with genital warts ( 0 vs. $13 \%$ ) - all diseases related to HPV infection. They also were more commonly diagnosed with candidiasis than were prevalent cases (40 vs. 17\%). Other microflora shown in Table 3 were not different in frequency between the two case groups. Incident cases were less likely to have prediagnostic symptoms of vulvar burning (79 vs. $94 \%$ ) or itching (57 vs. $73 \%$ ), clitoral pain

Table 4 WS-related symptoms prior to diagnosis or clinic visit

\begin{tabular}{lcccc}
\hline Symptoms & Cases n (\%) & Controls n (\%) & p-value & Age-adjusted OR (95\% Cl) \\
\hline Constant: never better* & $3(4.4)$ & & - & - \\
Constant: better, but persist* & $29(42.7)$ & & - & - \\
Intermittent* & $36(52.9)$ & & - & - \\
Vulvar burning & $63(91.3)$ & $8(12.3)$ & $<0.000$ & $73.4(23.7-227)$ \\
Vulvar itching & $47(68.1)$ & $15(23.1)$ & $<0.0001$ & $6.8(3.1-14.6)$ \\
Dysuria & $37(53.6)$ & $12(18.5)$ & $<0.0001$ & $4.9(2.2-10.8)$ \\
Frequent urination & $31(44.9)$ & $12(18.5)$ & $<0.001$ & $3.6(1.6-8)$ \\
Urgent urination & $32(46.4)$ & $9(13.9)$ & $<0.0001$ & $5.3(2.2-12.4)$ \\
Clitoral pain & $29(42)$ & $1(1.5)$ & $<0.0001$ & $45.7(6-350)$ \\
Rectal pain & $9(13)$ & $5(7.7)$ & 0.31 & $1.8(0.6-5.7)$ \\
Dyspareunia & $56(81.2)$ & $10(15.4)$ & $<0.0001$ & $23(9.3-57)$ \\
\hline
\end{tabular}

*Questions appropriate for WS only; $\mathrm{Cl}$, confidence interval; OR, odds ratio; WS, vulvar vestibulitis syndrome 
(29 vs. $46 \%$ ) or rectal pain (7 vs. $15 \%$ ). There was no difference in reporting dyspareunia (85 vs. $83 \%$ ) or dysuria (50 vs. $56 \%$ ).

\section{DISCUSSION}

This investigation found that VVS patients had a history of genital infections and diseases significantly more often than a group of healthy women. It is unlikely that these differences can be explained strictly on the basis of selection bias since almost all solicited women agreed to participate in the study. The review of medical and pathology reports to verify statements minimized any recall bias of patient self-reports. However, because lab results were not available for every event, it would be important to clarify whether these infections occurred at a different frequency in newly diagnosed (incident) cases, whether infection rates were higher in VVS because most were prevalent cases reporting rates based on both initial infections as well as subsequent events, and whether the frequency of infections was truly higher than reported by asymptomatic controls. In this study we found that most microflora and genital conditions were not reported at a different frequency between the incident and prevalent case groups, suggesting there was not bias in reporting more infections among prevalent cases. Physician bias associated with knowledge of a prior VVS diagnosis may have led to a higher frequency of evaluation and diagnosis of microflora in previous studies. We were not able to examine this issue in our cross-section of cases and controls but further assessment, by following diseased and healthy women and periodically performing the same clinical and laboratory assessments, is warranted.

Future epidemiologic research should evaluate risks in incident cases and follow them at subsequent intervals to characterize the natural history and risks of the disease. It is unknown whether there are differences in etiology between those who recover from VVS and those who continue to have symptoms. Based on the frequent lack of early diagnosis of VVS, patients may spend several years with the disease prior to a definitive diagnosis. Because of the long average duration of symptoms and the nature of the condition as one of unspecified persistence, prevalent cases that have been symptomatic for some time - regardless of the date of diagnosis - may represent groups with different etiologic risk factors. Prevalent cases of VVS also may have differential recall of symptoms because they have them more often over the course of the disease than newly diagnosed cases. Our investigation found that prevalent cases did recall a higher frequency of a number of VVS-related symptoms although not those that occur most commonly in incident cases, dyspareunia or dysuria.

An unexpected finding in this study was the frequency of vulvar dysplasia among VVS patients in contrast to its complete absence in controls. All were diagnosed at the time of VVS diagnosis by the same study gynecologist (Rudolph Galask). The incidence of VIN is low $\left(3 / 10^{5}\right)$ among those below the age of 35 , the age group considered to be at highest risk of VVS ${ }^{18}$. Seven of the VVS patients had a history of dysplasia at the time of study enrollment; thus over 40 patients/year would be expected to be diagnosed with VVS/vulvar dysplasia simultaneously at the vulvar clinic. That translates into $10 \%$ of cases of VVS cases having a diagnosis of vulvar dysplasia per year - women who might have developed cancer if not for their VVS symptoms. In support of a possible causal association, the presence of HPV oncogenic types is associated with risk of VIN in younger, but not older, women ${ }^{19,20}$. Since all the patients in our investigation were treated prior to testing for HPV, the lack of association between the current HPV result and vulvar dysplasia was not unexpected. It is possible that HPV is a risk for both VIN and VVS. In previous studies, the association between VVS and HPV has been inconsistent, with detection in between 5 and $100 \%$ of cases $^{6-11}$. Whereas Prayson and colleagues ${ }^{6}$, in a study of 36 women with vestibulitis, revealed no positive results for HPV types $6,11,16,18,31$ or 35 using DNA in situ hybridization, Costa and colleagues ${ }^{21}$ detected $80 \%$ of cases with HPV 16/18 (oncogenic viral types) using Southern blot. Bornstein and colleagues ${ }^{8}$ detected HPV in $54 \%$ of cases but only $4 \%$ of controls, using PCR/hybridization to detect HPV 6, 11, 16, 18 and 33, most of which were HPV 16 and 18. When examining other risks and confounders of VVS, comparing the $\mathrm{HPV}^{+}$and $\mathrm{HPV}^{-}$cases, they found no differences in $\mathrm{OC}$ use, parity, age, education, smoking or medically 
related diseases. Most recently, Morin and colleagues $^{22}$ detected HPV in $29.6 \%$ of vestibulitis cases and $23.9 \%$ of controls by PCR/hybridization capture assay - rates that are similar to those found in this study. Further, 17\% of the cases and 16.1\% of controls in their study had high-risk HPV whereas we detected oncogenic types in $14.5 \%$ of VVS and $18.5 \%$ of healthy women. The wide range of HPV frequency found in different studies may be explained by failure to include a control group, to include a larger number of older women, to use different, less sensitive lab techniques or to include a wide variety of HPV oncogenic types for assessment. Ideally, detection of HPV infection should be performed prior to treatment for dysplasia or VVS and then subsequently reevaluated in conjunction with disease status and symptoms.

It is unclear why the healthy controls in this study had much higher rates of two other diseases, ASCUS and cervical dysplasia, since they were recruited from private staff practices, not high-risk clinics. HPV infection is associated with only a small portion of those who are diagnosed with these diseases and may explain why there was no association between a history of these conditions and current HPV status. Treatment also may explain this lack of association. Although HPV has been linked to VVS, there have been no previous reports of an association between these cervical conditions and VVS disease. This inconsistent association may be due to greater vulnerability of the epithelia of women with vestibulitis to (HPV) infection rather than a causal association between HPV and VVS.

The increased prevalence of other genital infections, including yeast infections ${ }^{12}$, candidiasis ${ }^{3,12,23}$, trichomoniasis ${ }^{12}, \mathrm{BV}^{12}, \mathrm{PID}^{12}$ and genital herpes ${ }^{12}$, has been found in some but not all previous investigations of microflora and vestibulitis. A history of candidiasis is one of the more consistent infections detected at a higher rate in these women. Sarma ${ }^{12}$, in a small case-control study, also reported $\mathrm{BV}$ and yeast infections more frequently in VVS patients but not trichomoniasis or PID as found in this investigation. One possible explanation for the differences in findings may be attributable to our review of prior medical and pathology lab results or referring physician statements of genital infections to corroborate patient reports of infections and diseases. Other conditions examined in this study - vaginal dysplasia, gonorrhea and syphilis - have not been characterized in other research. Based on our data, these would appear to present no risk in association with vestibulitis.

Although our VVS cases were somewhat younger on average than were controls, both the average and range of ages were comparable to reports found in other studies ${ }^{5,6}$. There were no significant changes in findings for demographics, sexual behavior, genital diseases or symptoms between crude and reported age-adjusted rates. Because VVS was more frequently diagnosed with increasing income, the characteristics of the patient population may be biased towards those who have greater financial access to health care. However, educational level, a more significant indicator of health care behavior, did not differ between cases and controls. Our VVS patient population mirrors that of other studies in regard to race, namely that Caucasians are the predominant racial group to present with this condition ${ }^{1,12}$. Whether this is a reflection of socioeconomic status or differences in predisposition to risk is unclear. The fact that a higher frequency of VVS patients were married, currently having sex and using OCs may be indicative of a greater risk of exposure to STDs. Compared with healthy women, those with VVS did indicate significantly higher rates of genital discomfort and pain, particularly dyspareunia $(81$ vs. $15 \%, p<0.0001)$ and clitoral pain (42 vs. $2 \%$, $p<0.0001)$. These findings related to sexual behavior also contradict the belief that patients with this condition avoid sexual contact because of a high rate of pain ${ }^{1,5}$. It must be remembered, however, that the average duration until diagnosis in the VVS group was 2 years and several more years until the time of the study, by which time a large percentage of patients noted that their symptoms were better or intermittent.

Several studies have postulated that the underlying factor important in the development of this disease is related to the immune system ${ }^{14}$. An alteration in the immune system of VVS patients is supported not only by the higher risk of genital infections but also that of URIs, immunodeficiency diseases, and musculoskeletal problems, 
also identified in this and other studies ${ }^{12,24,25}$. Ashman and $\mathrm{Ott}^{23}$ hypothesized that autoimmunity may play a role in vestibulitis risk. They propose that in susceptible individuals, antigens of candida are cross-reactive with certain vulvovaginal tissue antigens, thus preventing an effective immune response, and that after repeated infections the immune system becomes hyper-reactive against these cells. These responses could be triggered by hormonal changes or use of OCs. In support of this hypothesis, Bornstein ${ }^{8}$ found an association between vestibulitis and use of OCs. Our case population also had a higher rate of candida infection, current use of OCs and use of other sex steroid hormones.

Biopsy studies of tissue from women with vestibulitis have shown local chronic inflammatory reactions ${ }^{6}$. Foster and colleagues ${ }^{26}$ demonstrated that vulvar biopsies from women with vestibulitis also had higher levels of interleukin-1 (IL-1) and tumor necrosis factor alpha (TNF $\alpha$ ) than controls. These findings are consistent with the data of Chadha and colleagues ${ }^{27}$ who examined punch biopsies from the vulvar vestibule of VVS cases and age-matched controls. They found chronic inflammatory infiltrate present in all specimens with VVS, which consisted primarily of $\mathrm{T}$ lymphocytes and a small percentage of $\mathrm{B}$ cells, plasma cells and mast cells. In a preliminary study evaluating sera for NK cell activity to determine whether vestibulitis patients had alterations in immune regulation ${ }^{22}$, we found that overall, cases had significantly decreased NK cell activity compared with age-matched controls, with an average lytic level of 0.93 in VVS cases and 4.19 in controls. While it is not possible to determine whether the NK cells were decreased as a result rather than a cause of the disease, one reasonable interpretation is that the cells migrated to the tissue to combat infections.

Based on our findings and other clinical and immunologic studies, there is further need to examine immunological parameters in both the blood and affected tissue to correlate findings with the findings of various microfloral lab results. To better understand etiologic and preventive mechanisms of vestibulitis, potential microfloral risk factors and immunologic markers need clarification in a group of diagnosed VVS cases and healthy controls who receive similar diagnostic work-up and who are followed over time for changes in infection status, symptoms and recovery.

\section{REFERENCES}

1. Bergeron S, Blinik YM, Khalife S, Pagidas K. Vulvar vestibulitis syndrome: a critical review. Clin J Pain 1997;13:27-42

2. Freidrich EG. Vulvar vestibulitis syndrome. J Reprod Med 1987;32:110-14

3. Woodruff JD, Parmley TH. Infection of the vulvar vestibular gland. Obstet Gynecol 1983;62: 609-12

4. Vulvodynia Workshop. Current knowledge and future directions. Workshop Proceedings. NIH, 1997

5. Pagano R. Vulvar vestibulitis syndrome: an often unrecognized cause of dysparenunia. Aust NZ J Obstet Gynecol 1999;39:79-83

6. Prayson RA, Stoler MH, Hart WR. Vulvar vestibulitis. A histopathologic study of 36 cases, including human papillomavirus in situ hybridization analysis. Am J Surg Pathol 1995;19:154-60

7. Bornstein J, Shapiro S, Rahat M, et al. Polymerase chain reaction search for viral etiology of vulvar vestibulitis syndrome. Am J Obstet Gynecol 1996; 175:139-44
8. Bornstein J, Shapiro S, Goldschmid N, et al. Severe vulvar vestibulitis. Relation to HPV infection. J Reprod Med 1997;42:514-18

9. Sjoberg I, Lundqvist EN. Vulvar vestibulitis in the north of Sweden. An epidemiologic case-control study. J Reprod Med 1997;42:166-8

10. Bazin S, Bouchard C, Brisson J, et al. Vulvar vestibulitis syndrome: an exploratory case-control study. Obstet Gynecol 1994;83:47-50

11. Origoni M, Rossi M, Ferrari D, et al. Human papillomavirus with co-existing vulvar vestibulitis syndrome and vestibular papillomatosis. Int $J$ Gynecol Obstet 1999;64:259-63

12. Sarma AV, Foxman B, Bayirli B, et al. Epidemiology of vulvar vestibulitis syndrome: an exploratory case-control study. Sex Transm Infect 1999;75: 320-6

13. Boehm-Starke $\mathrm{N}$, Hilliges $\mathrm{M}$, Falconer C, Rylander E. Increased intraepithelial innervation in women with vulvar vestibulitis syndrome. Gynecol Obstet Invest 1998;46:256-60 
14. Masterson BJ, Galask RP, Ballas ZK. Natural killer cell function in women with vestibulitis. $J$ Reprod Med 1996;41:562-8

15. Summersgill KF, Smith EM, Kirchner HL, et al. p53 polymorphism, human papillomavirus infection, and oral cancer. Oral Surg Oral Med Oral Pathol Oral Radiol Endod 2000;90:334-9

16. Haldane JBS. The estimation and significance of the logarithm of a ratio of frequencies. Ann Hum Genet 1955;20:309-11

17. SAS system for Windows. Version 8.0. Cary: SAS Institute, 1999

18. SEER Stat3.0. NCI. Silver Springs: Information Management Services, 2000

19. Trimble CL, Hildeshiem A, Brinton LA, et al. Heterogeneous etiology of squamous carcinoma of the vulva. Obstet Gynecol 1996;87:59-64

20. Madeleine MM, Daling JR, Carter JJ, et al. Cofactors with human papillomavirus in a population-based study of vulvar cancer. $J$ Natl Cancer Inst 1997;89:1516-23

21. Costa S, Rotola A, Terzano P, et al. Is vestibulitis papillomatosis associated with human papillomavirus? J Med Virol 1991;35:7-13

ReCEIVED 09/24/01; ACCEPTED 02/25/02
22. Morin C, Bouchard C, Brisson J, et al. Human papillomaviruses and vulvar vestibulitis. Obstet Gynecol 2000;95:683-7

23. Ashman RB, Ott AK. Autoimmunity as a factor in recurrent vaginal candidosis and the minor vestibular gland syndrome. J Reprod Med 1989;34: 264-7

24. Kollias G, Douni E, Kassiotis G, Kontoyiannis D. The function of tumour necrosis factor and receptors in models of multi-organ inflammation, rheumatoid arthritis, multiple sclerosis and inflammatory bowel disease. Ann Rheum Dis 1999; 58:132-9

25. Kline JN. Effects of CpG DNA on Th1/Th2 balance in asthma. Curr Top Microbiol Immunol 2000;247:211-25

26. Foster DC, Hasday JD. Elevated tissue levels of interleukin-1 beta and tumor necrosis factor-alpha in vulvar vestibulitis. Obstet Gynecol 1997;89:291-6

27. Chadha S, Gianotten WL, Drogendijk AC, et al. Histopathologic features of vulvar vestibulitis. Int J Gynecol Pathol 1998;17:7-11 


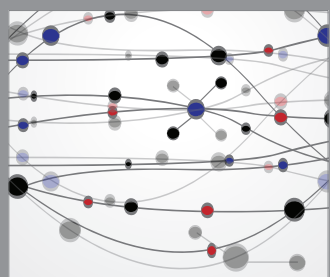

The Scientific World Journal
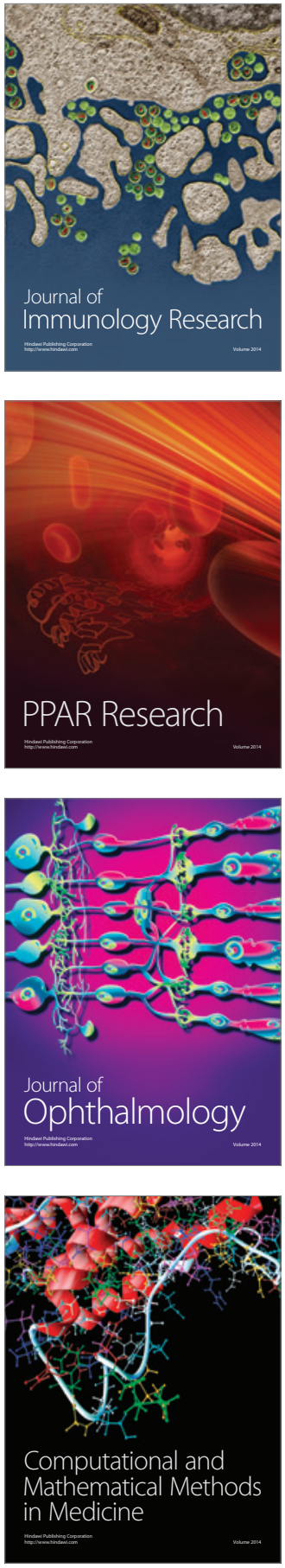

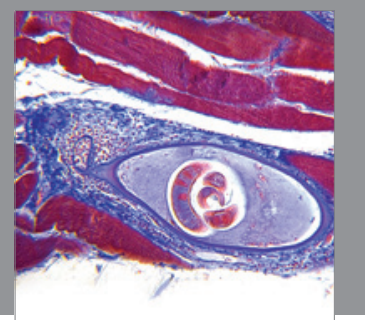

Gastroenterology

Research and Practice
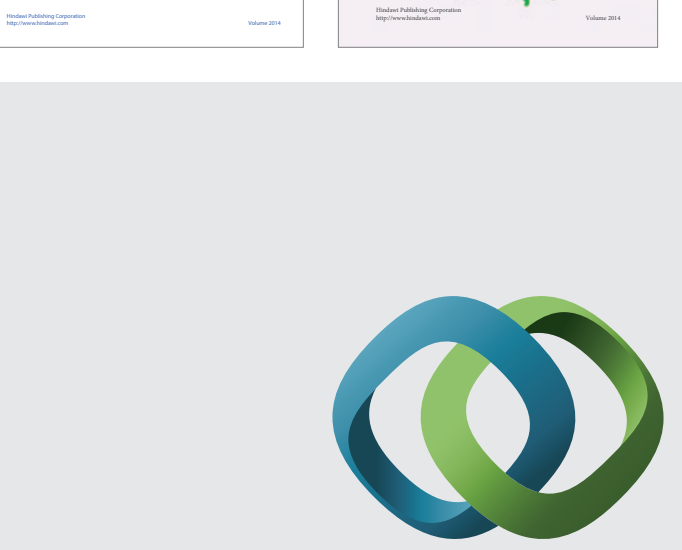

\section{Hindawi}

Submit your manuscripts at

http://www.hindawi.com
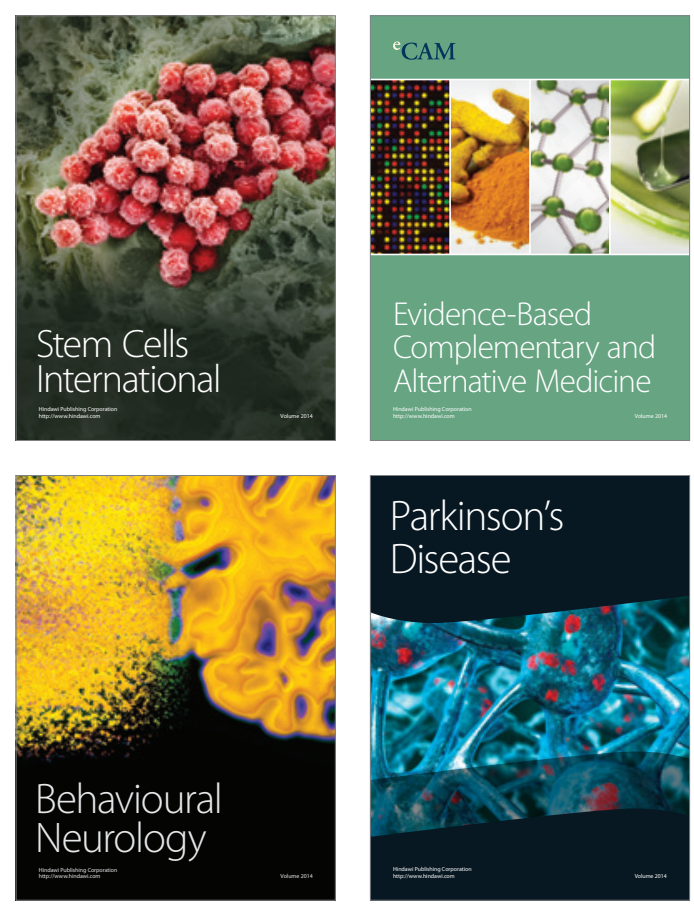

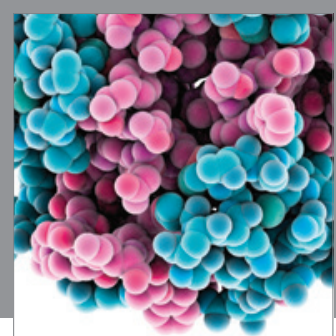

Journal of
Diabetes Research

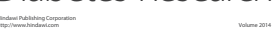

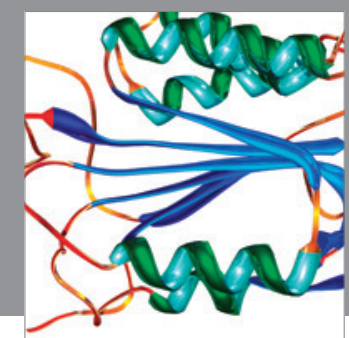

Disease Markers
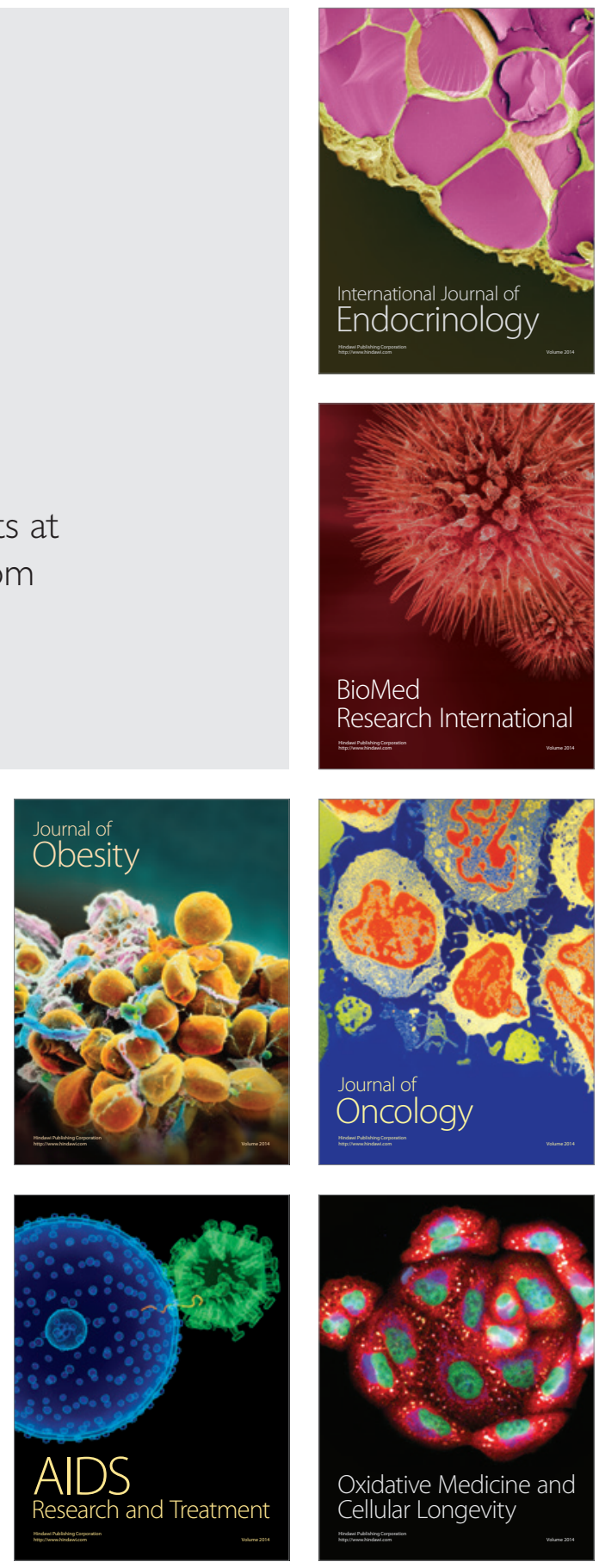